DETC2008-49799

\title{
DESIGN ACHIEVEMENT MODEL FOR PLANNING CREATIVE AND CONCURRENT DESIGN PROCESS
}

\author{
Yutaka Nomaguchi, Daisuke Tsutsumi and Kikuo Fujita \\ Department of Mechanical Engineering \\ Osaka University \\ Suita, Osaka 565-0871, JAPAN \\ Email: noma@mech.eng.osaka-u.ac.jp \\ Tel: +81-6-6879-7324, Fax: +81-6-6879-7325
}

\begin{abstract}
Planning of an upstream design process that includes creative and concurrent activities has become more important for product development in a competitive market. A significant characteristic of upstream planning is that the design process is one of knowledge creation. During this process, a designer makes progress toward a more advanced knowledge level that corresponds to a more advanced design achievement. In most cases of a creative and concurrent design process, however, a designer has to compromise design achievement because of constraints such as delivery time, cost, and another designer's intention. Therefore, in planning a design process, it is more essential to set an acceptable level of design achievement and to predict whether or not a planned design process can ensure this level, than to predict the design time needed to totally achieve the design goals. This paper proposes a new method of design process planning that focuses on quantitative prediction of knowledge level achieved in a creative and concurrent design process. A growth curve model using fuzzy numbers is introduced to predict the final achievement of each task and final achievement of consistency between tasks after running a planned design process. The reliability model of a serial system is used to calculate the total acceptability of the design achievement. An experimental system that supports design process planning based on the proposed method is developed. This paper demonstrates its application to a student design
\end{abstract}

project in order to show the power of the method.

Keywords: Design process planning, design achievement, design structure matrix, growth curve, fuzzy number, reliability model

\section{INTRODUCTION}

Manufacturers engaged in product development face a number of challenges, such as dealing with increasingly complicated systems, sophisticated components, diversifying product variety, and shortening lead times. Upstream planning of the design process that includes both creative and concurrent activities has become more important for product development efforts in a competitive market. A manufacturer must define appropriate tasks which comprise the design process and appropriately allocate designers, time and budget to these tasks. However, the upstream portion of the design process is rather indistinct. Often the accomplishment of each task cannot be discriminated by a threshold, and design achievement is not always clearly measurable. This means that upstream planning requires not only relative or qualitative analysis such as the DSM (Design Structure Matrix) method, but also quantitative analysis that models indistinct design process factors.

The upstream design process is characterized by knowledge creation. A designer makes progress toward more advanced knowledge levels that correspond to more advanced design 
achievement. A designer does not only apply alreadysystematized knowledge such as knowledge about physics, but also progressively acquires concrete knowledge on the whole and parts of a product while exploring what general knowledge to apply and how to apply it (Schön, 1982). In a concurrent design process with collaboration of many designers, knowledge is acquired through a spiral transformation among tacit/explicit and group/individual knowledge (Nonaka and Takeuchi, 1995). Although performance depends on several factors, including each designer's skill, the collaboration mechanism used by the designers, etc., such acquisition is accomplished gradually as the design process progresses. This research focuses on this "progressive nature of knowledge acquisition" as an important viewpoint in planning a creative and concurrent design process (Nomaguchi et al., 2007).

This paper proposes a new planning method for a creative and concurrent design process that focuses on quantitative prediction of achieved knowledge level that corresponds to a design achievement. A growth curve model that uses fuzzy numbers is introduced to predict the final achievement of each task and final achievement of consistency between tasks after running a planned design process. The reliability model of a serial system is used to calculate total acceptability of design achievement. An experimental system that supports design process planning based on the proposed method is developed. This paper demonstrates its application to a student design project in order to show the power of the method.

\section{PLANNING OF CREATIVE AND CONCURRENT DESIGN PROCESS}

\subsection{A Viewpoint of Knowledge Creation}

While any design process includes some aspects of knowledge creation activity, it is more characteristic of the upstream design process. When such a design process is planned, a design process manager should consider that the progress of knowledge acquisition must be cut off at the delivery time, since the design effort is not directed at perfect optimizing but at satisfying a specific problem. A designer has to compromise the design achievement because of various design constraints such as delivery time, cost, and other designer's intentions, and so on. Therefore, in the planning of a creative and concurrent design process, it is more essential to set an acceptable level of design achievement and to predict whether or not a planned design process ensures this level, than to predict the design time that is needed for totally achieving the design goals. The objective of such a design process planning is to maximize the possibility of a higher achievement level of knowledge.

The ability of knowledge acquisition, that is, the ability to achieve a successful design, depends on how much prerequisite knowledge a designer has, how experienced a designer is in the assigned task, and so forth. A design process manager should schedule a superior designer, who has good knowledge acquisition abilities, to perform critical tasks for achieving the above objective. The choice of communication mechanism also influences knowledge acquisition capabilities. As today's scale of product development is not one of any simple tool, product development is usually executed by a collaboration of multiple designers, or sometimes, collaboration of multiple teams since the amount of knowledge acquisition is divided and assigned to a group of designers and teams. In other words, a design process is divided into specific tasks, each designer or team takes charge of a portion of these tasks, and the team is coordinated toward better collaboration. Designers or teams are required to communicate with each other in order to acquire design knowledge and to secure consistency among the distributed tasks. While such communication is indispensable, too much communication often makes the overall design process inefficient. Thus, stronglyrelated tasks should be assigned to a single designer or a single team to relax communication costs, because communication within a smaller group is more efficient than communication within a bigger group or communication between different teams.

\subsection{Related Work}

This subsection briefly surveys design process planning approaches and methods that have been proposed, and discusses the open issues in planning creative and concurrent design processes.

2.2.1 Task and iteration identification The first phase of design process planning is to identify and describe tasks and their iterations. There are several methods for this phase of process planning. For example, IDEF0 (Marca, 1988) is one of the most conventional approaches. It is designed to make explicit the decisions, actions, and activities of an organization or system by input and output of information. DSM is also widely recognized as a method for modeling iterations of the design process (Eppinger, 1994). DSM represents a the dependency among tasks by a matrix. Clustering algorithms that group related tasks together and partitioning algorithms that permute tasks so as to reduce possible iterations are proposed under the DSM scheme. IDEF0 and DSM provide an at-a-glance description of the design process, and allow a design process manager to arrange a structure of iterations. These approaches are most effective downstream in the design process, because reducing iterations is very critical in planning. However, iteration is useful or often inevitable in upstream planning because of the progressive nature of knowledge acquisition. A design process manager should consider how much iteration is needed in order to achieve a target knowledge level. This means that planning of such upstream design requires not only iteration identification, but also quantitative analysis of design achievement. 


\subsubsection{Quantitative prediction of design process} factors Quantitative estimates of design quality and lead time of an upstream design process are uncertain and may present a bottleneck, because they depend on indistinct factors, such as the skill of an individual designer, the collaboration mechanism of designers and so on. A good design manager can carry out such a design process planning by empirically understanding the indistinct factors of the design process. However, as the number of participating designers and the scale of the design process increase, it obviously becomes more difficult for even very good managers to generate superior and appropriate design process plans without any evaluation of the plan's quantitative aspects. To rationally support planning of a creative and concurrent design process, any methodology for evaluating quantitative features is as indispensable as theory or procedure. Some methodologies for quantitative evaluation of the design process have been proposed in recent years, and they have introduced many mathematic models for describing the occurrence of task iterations somehow.

Chao et al. have proposed Design Task QFD that represents relationships between risk factors of design and tasks and supports estimation of the magnitude of risk for each task (Chao and Ishii, 2003; Chao and Ishii, 2004; Chao and Ishii, 2007). Ostergaard et al. proposed a methodology that evaluates the efficiency of collaboration in a design process by using an electric circuit analogy (Ostergaard and Summers, 2007). This methodology models risk factors of collaboration as resistance in the electric circuit so as to quantitatively estimate collaboration efficiency. Yang et al. proposed a methodology of risk estimation based on gain analysis of a decision network (Yang et al., 2005).

Simulation-based approaches are also available to estimate uncertainty. A rework simulation (Cho and Eppinger, 2005; Yassine et al., 2001) is typical of these approaches. In this simulation, rework probability is defined for iteration between tasks that is represented by DSM. The Monte Carlo method can be adopted for estimating the duration of a design process. A research group of Clarkson has been developing Signposting methodology, using a Markov chain-based simulation method (Clarkson and Hamilton, 2000; Melo et al., 2002; Jarrett et al., 2002; O'Donovan et al., 2004; Wynn et al., 2005). The Signposting method defines a task as a state transition that changes the values of design parameters. The design process is defined as a chain of state transitions. Transition probability is defined for each state transition so as to estimate the duration, cost and design quality of the whole design process, as well as the ranges of these parameters.

\subsection{Our Approach}

Although many researchers have tackled this problem, we do not yet have a definitive model of a design process, especially one which is effective for the upstream phase of a creative and concurrent design process, because of difficulty in modeling the aforementioned indistinct factors. We present the hypothesis that the progressive nature of design achievement is a key to establishing a design process model, and for this reason we adopt an axiomatic approach for formulating the design process to exclude vague factors as much as possible with this reason (Nomaguchi et al., 2007). Even though such a mathematical approach has some shortcomings in the aspects of describing detailed characteristics, it is expected to be robust against the indistinct factors and to be self-consistent within the theory.

This research develops a planning method of a creative and concurrent design process, and its associated planning tools based on the formulated definitions and models. In order to formulate a planning method, the next section analyzes a student design project and reveals some essential factors of a creative and concurrent design process from the viewpoint of progressive nature of knowledge acquisition.

\section{ANALYSIS OF CREATIVE AND CONCURRENT DESIGN PROCESS}

\subsection{A Case Analysis of a Student Design Project}

A case analysis of this research was executed by intensive interviews with the OFRAC (Osaka university Formula RAcing Club) members.

The Student Formula SAE Competition of Japan (Formula SAE, 2008) was started in 2003 to provide an opportunity for students to develop their engineering skills. OFRAC is the team of Osaka University that has entered this competition since its beginning. While a student project to design a formula racing car is not the same as a project taking place at a manufacturing firm, it is small-sized but as highly creative and collaborative as an engineering design project. It contains all phases from conceptual design to manufacturing design. In addition, all information is open and easily accessible for the university members. With these reasons, the OFRAC design project is used as a case for investigating the nature of the design process.

Figure 1 shows the formula car designed and built by the OFRAC project in 2006, along with its major specifications. About twenty undergraduate and graduate engineering students of Osaka University participate yearly in the project, and they design, manufacture, and assemble almost all of the components except for some parts such as major components of its power train, which are provided by a vehicle manufacturer. The members of the project have changed every year because of student graduation and entrance. They have tackled new engineering challenges every year while inheriting the design and results of the previous year. This means that some members are novices and others are experts. Since the knowledge level is diverse among them, consistency of their knowledge acquisition must be secured through several types of team meetings directed toward design creativity and engineering challenges. 


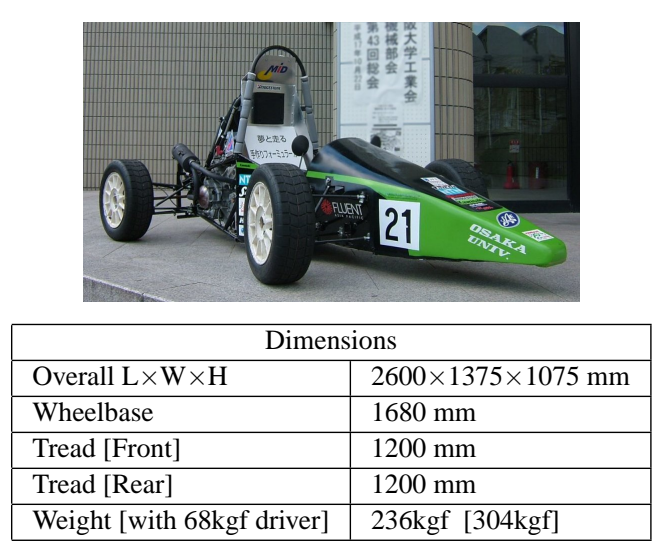

\begin{tabular}{|l|l|}
\hline \multicolumn{2}{|c|}{ Suspension Parameters [Front \& Rear] } \\
\hline Suspension Type & Double wishbone \\
\hline Tire size & Racing slicks 20.0x7.5-13 \\
\hline Wheels & 13 in, 5.5J, Offset +45, Hole 4, PCD \\
& 100 \\
\hline Minimum ground & $80 \mathrm{~mm}$ \\
\hline & Power train \\
\hline Engine & Model 609cc KAWASAKI KVF-650 \\
& CVT \\
\hline Compression ratio & $10.4: 1$ \\
\hline Induction & NA \\
\hline Fuel type & High-octane gasoline \\
\hline Max power RPM & More than 30.3PS/4000 \\
\hline Max torque RPM & More than 6.5kgf-m/3500 \\
\hline Fuel system [manf'r] & FI \\
\hline
\end{tabular}

Figure 1. Formula car of OFRAC

Figure 2 shows the DSM of this project, which was based on the collected task information and progress reports. Because the architecture of a formula car is not so variable, a task of this project corresponds to a single component, such as the braking system or the car's frame. Each task contains a conceptual design phase, a detailed design phase and a manufacturing phase. In general, a design project sometimes begins with identifying the tasks. While task identification should be a part of an integrated planning methodology, this paper focuses on scheduling and assigning designers to identified tasks. In the matrix, a dependency of a task is represented with four numbers, $0,1,3$, and 9 , according to the degree of interactions between specific tasks. The larger the degree, the stronger the dependency. This matrix shows that the project is divided into 27 tasks, and that these tasks are allocated to four teams. As shown in the matrix, there are various types of interactions, that is, some are within a team, and others are across different teams. Further, more interactions are allocated within each team in order to efficiently and effectively share and exchange knowledge.

\subsection{Factors of Design Process Planning}

Our analysis of the student design project yields the following observations (Nomaguchi et al., 2007);

- Design quality of a task corresponds to the knowledge level which an assigned designer acquires by executing a task.

- Consistency between tasks corresponds to the knowledge level which designers assigned to the tasks have acquired through communication.

- Communication within a team is more effective than communication between teams.

- The fewer tasks concerned with communication, the more effective the communication will be.

These extracted facts forms the basis for formulating and developing a model which is explored in the next section.

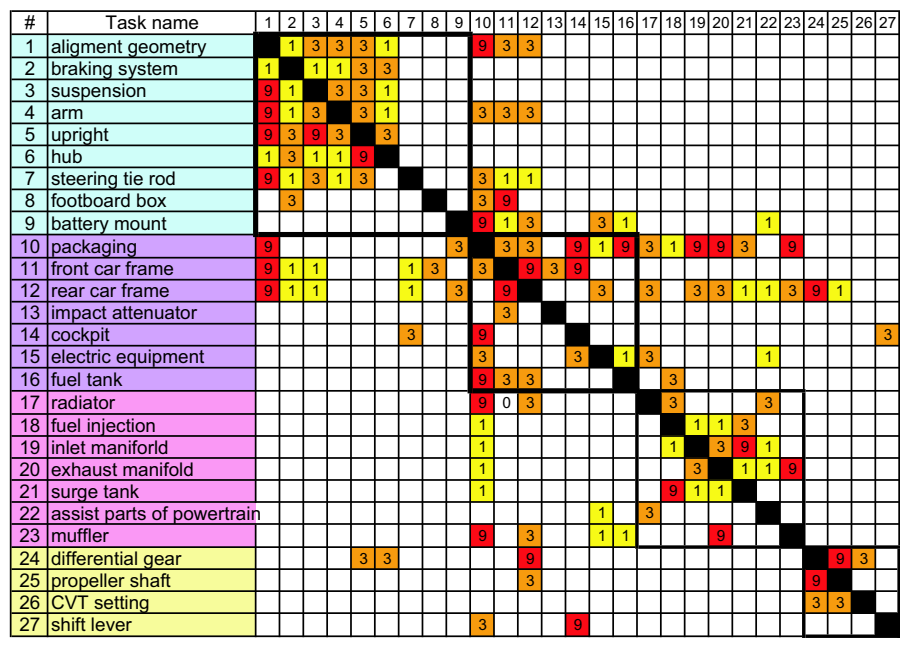

Figure 2. Design Structure Matrix of the formula car project

As stated, they strongly depend on human factors, such as individual character, private life influences, relationships between designers, individual motivations, and so on. This research limits minimizes the set of indistinct factors in design process planning, and it is expected to be robust against the indistinct factors and to be self-consistent within a theory.

The following factors are defined in order to discuss a design process planning method.

3.2.1 Task This research defines a task as a part of a design process. As the scale of recent product development efforts has become larger, the required amount of knowledge has become massive. It is usual that a product development is done by collaboration of multiple designers, or sometimes, collaboration of multiple teams. In this collaboration, a designer 
takes charge of a certain part of the design process, that is, a task. Most tasks are usually defined as a part of the design process that corresponds to a part of a product. In the student design project, a braking system design, a suspension design, etc. are defined as a task within the formula car design.

3.2.2 Design achievement, knowledge level Design achievement is the degree of reliability of the designed product's performance. This research assumes that design achievement corresponds to the level of knowledge which a designer acquires through the design process. A designer can acquire knowledge at an advanced level through design activities and learning based on knowledge at a fundamental level that he/she has already acquired.

This progressive nature of knowledge acquisition can be found in the following example of a formula car design. The performance of a car frame is measured in terms of light weight and rigidity to some collision modes. Therefore, a designer who is in charge of a car frame should acquire knowledge for this task from a textbook on the strength of material and fracture mechanics. He or she can perform a more detailed investigation by means of a structural CAE analysis. However, it is necessary to acquire knowledge in order to understand a car frame at a more advanced level. For instance, an OFRAC member has come to understand that the rigidity of the frame connection part is more important than the rigidity of the frame material in evaluating the overall rigidity of the frame, and that the rigidity of the connection between a front arm and a bell crank is the most important. This means that a designer should consider the modulus in the torsion of the connected part when he or she builds a quantitative model of the frame rigidity. These important facts have been understood through actually producing the formula car. It is difficult to obtain this kind of knowledge without design practice. Although the performance of such acquisition depends on each designer's skill, the collaboration mechanism of designers, etc., it is observed that every project member shows such progress. However, this progress of knowledge acquisition must be cut off at the delivery time. In the case of OFRAC project, members must finish the design process in 84 days in order to meet a deadline of making an entry into the competition. It is important for the design process manager to assign designers and teams carefully in order to achieve the target level of each task.

\subsubsection{Task dependency, task consistency Since} each part of a product depends on other part(s), each task also depends on other task(s). Therefore, a designer has to carry out a task while taking care of consistency with other task(s). This research defines task dependency as the degree of dependency of two tasks that can be measured by how much knowledge of task 2 is required to carry out task 1 . Task consistency is defined as a degree of consistency between tasks. For example, a task of car frame design deeply depends on other tasks, such as cockpit design, as shown in Figure 2. A designer of a car frame should frequently communicate with the designer of the cockpit in order to enhance the consistency between the components and rationally carry out the tasks.

3.2.4 Concurrent design process Designers should frequently communicate with each other in order to rationally carry out depending tasks. However, a designer cannot always acquire the latest information of the other tasks at any time. A designer should carry out the task by setting some assumptions about the part concerning the other tasks. This means that tasks are often done in a concurrent way even if the tasks depend on each others.

Such a situation can be also explained with the example of the student formula car project. The task of car frame design strongly depends on cockpit design. When the rigidity of the frame is evaluated, the designer needs information regarding cockpit position. Then, the designer of the car frame contacts the designer of the cockpit, and calculates the frame rigidity based on information obtained. On the other hand, the designer of the cockpit may make an effort to improve the cockpit design performance, such as visibility range of a driver, and may revise the position of the cockpit for that. The information of cockpit position will only be assumed at that time.

3.2.5 Meeting Meeting is defined as a part of the design process whereby a designer confirms consistency of his/her knowledge with the other task(s). By meeting with the other designers, a designer can know whether or not his/her knowledge should be revised. When knowledge is revised in a meeting, task achievement is reduced.

Let us continue where an example of a car frame design left off in Subsubsection 3.2.4. As for the frame rigidity calculation based on old information, when cockpit position has been revised, the rigidity calculation should be also revised. That is, the assumed knowledge becomes false at this occasion. The achievement level concerning the car frame design temporarily falls, and therefore, the designer should design again and improve the achievement level. However, the consistency between the car frame and the cockpit was improved by this revision.

\section{DESIGN ACHIEVEMENT MODEL}

\subsection{Overview of Design Achievement Modeling}

Based on the case study of OFRAC, this research models a design achievement prediction in order to formulate a planning method of a creative and concurrent design process. Firstly, this 


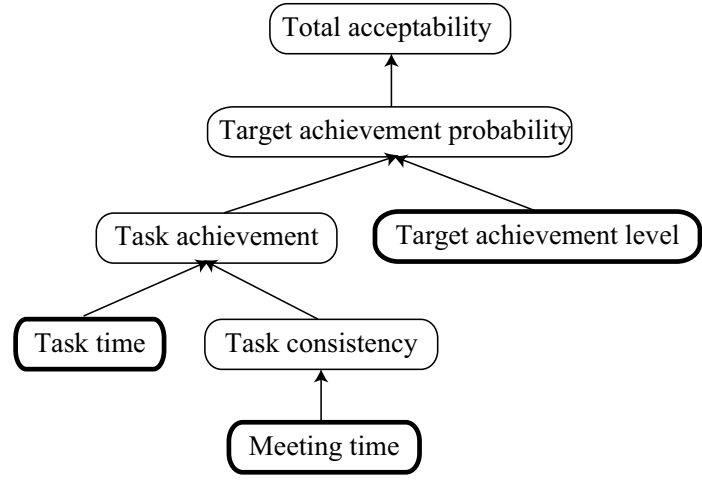

Figure 3. Overview of design achievement modeling

subsection briefly provides an overview of design achievement modeling.

Figure 3 shows relationships among the factors of a design process relating to design achievement evaluation. On the Figure, a node with a heavy line is a planning factor. A task achievement is predicted by a growth curve that is a monotonically increasing function of a task time. However, if the consistency among dependent tasks is low, a task achievement can be reduced. Task consistency is promoted by a meeting. A target achievement probability is calculated by how much a predicted level of task achievement surpasses a planned achievement level. Finally, the total acceptability of the design process is evaluated by multiplying the target achievement probability of all tasks.

\subsection{Quantification of Knowledge Level}

A quantitative scale of knowledge level is necessary in order to discuss design achievement quantitatively. Sakamoto and Fujita present a method for estimating the achievement level of the optimal design of an electronic product (Sakamoto and Fujita, 2006). For this purpose, they propose the following tendegree scale for quantifying design achievement level (Sakamoto and Fujita, 2006). This research uses this as a scale of design achievement level.

Level 0.0 : Relating knowledge of a task is unknown. This corresponds to the initial condition of totally new design.

Level 0.1: A designer can refer to a product of past generations or other companies that has a good track record in a market. This corresponds to the initial condition of similar design.

Level 0.2: A designer knows a qualitative model to evaluate design object.

Level 0.3: A designer knows a single quantitative model to evaluate design object. But the designer relies upon a qualitative understanding of conditions to apply the model.
Level 0.4: A designer knows a single quantitative model to evaluate the design object. The designer also knows quantitative conditions for applying the model.

Level 0.5: A designer knows multiple quantitative models to evaluate the design object. The designer also knows their conditions quantitatively.

Level 0.6: A designer knows a single-objective optimization model of the design object. But the designer relies upon a qualitative understanding of conditions to apply the model.

Level 0.7: A designer knows a single-objective optimization model of the design object. The designer also knows its conditions quantitatively.

Level 0.8: A designer knows a multi-objective optimization model of the design object of a product. But the designer uses a qualitative understanding of conditions for applying the model.

Level 0.9: A designer knows a multi-objective optimization model of design object. The designer also knows its conditions quantitatively.

Level 1.0: A designer knows a complete multi-objective optimization model of design object of a product. This is the limiting highest level.

A designer acquires knowledge through the design progress from knowledge at a fundamental level to that at an advanced level for a specific task.

\subsection{Growth Curve Model with Fuzzy Number}

A designer can advance his/her knowledge more quickly at a fundamental level than at an advanced level. In order to model this pace of knowledge level progress, Sakamoto introduced the following exponential growth curve model that is usually used in the prediction of a system's reliability (Ichikawa, 1990). This model represents the progress of knowledge level toward the highest level under the above scale of quantification;

$$
f(t)=1-\left(1-f_{0}\right) \exp (-m t)
$$

where, $t$ denotes design time, $f(t)$ is the knowledge level at time $t, f_{0}$ is the initial knowledge that a designer has at the beginning of the task, and $m$ is the difficulty of a task. $f_{0}$ depends on a designer, and $m$ depends on a task.

The research reported hear applies this model to the analysis of the formula car design project. A value of $f(t)$ was determined by asking a designer to describe the achievement level at a time point $t$ on a scale of ten. If achievement levels at two time points $f\left(t_{1}\right), f\left(t_{2}\right)$ are given, $m$ and $f_{0}$ can be determined. For example, a growth curve of the inlet manifold design task of the OFRAC project can be determined as follows. According to an interview with a designer who took charge of this task, he only knew a qualitative model of an inlet manifold at the beginning 


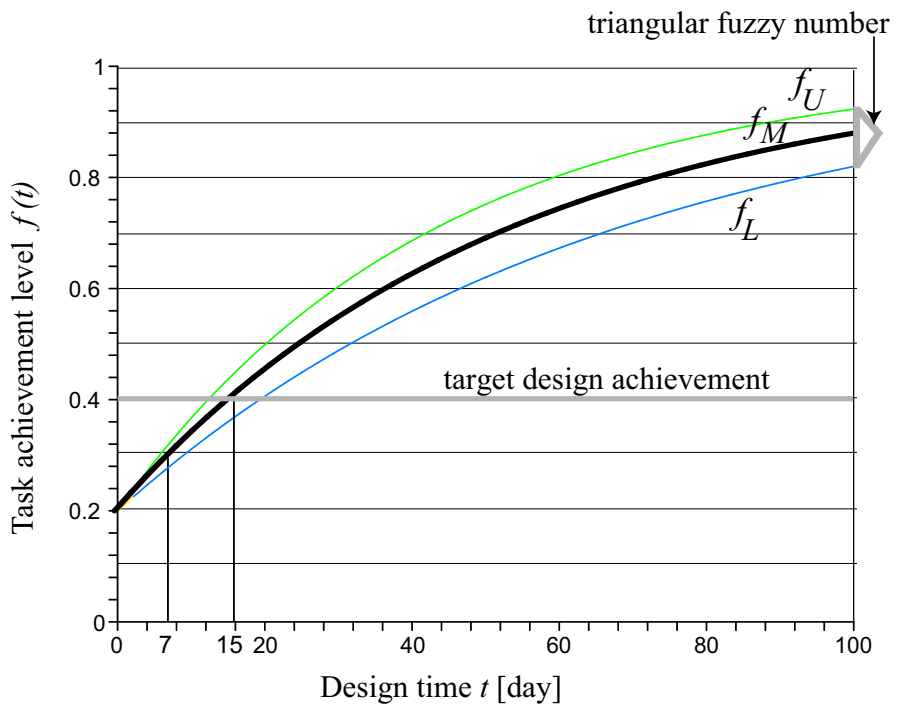

Figure 4. Growth curve of inlet manifold design achievement

of design. This corresponds to level 0.2. After seven work days, he had learned a quantitative model to evaluate an inlet manifold although he had not yet learned its conditions. This corresponds to level 0.3. The difficulty of this task $m$ can be solved by $f(0)=f_{0}=0.2$ and $f(7)=0.3$, then $m=0.019$. The heavy line of Figure 4 shows a growth curve of the inlet manifold design task. A task's necessary duration to reach the target achievement level can be estimated by using the above growth curve. When a target level of inlet manifold design is 0.4 , Figure 4 indicates that it will take about 15 work days. If a manager wants to leverage this task's achievement level, he/she will need to ask the designer to work more harder, or another designer, whose $f_{0}$ is larger than this designer's, should take charge of this task.

According to Sakamoto's case studies of electronic product design, this model gives a good prediction of design achievement. However, its prediction includes uncertainty because of uncertain parameters $f_{0}$ and $m$. Because task achievement levels $f\left(t_{1}\right), f\left(t_{2}\right)$ are given by one of ten levels when $m$ is determined, a given value of $f$ contains an error margin of $\pm 0.05=\varepsilon_{f}$. This research introduces a fuzzy mathematical model to the growth curve model in order to rationally represent and calculate its uncertainty. A triangular fuzzy number is represented by $A=\left(a_{L}, a_{M}, a_{U}\right)$, where $a_{L}$ is a lower point of a triangular distribution, $a_{M}$ is a middle point and $a_{U}$ is an upper point. A value of $m$ is represented by a triangular fuzzy number $\left(m_{L}, m_{M}, m_{U}\right)$, where $m_{L}$ is determined by $f\left(t_{1}\right)+$ $\varepsilon_{f}$ and $f\left(t_{2}\right)-\varepsilon_{f}, m_{M}$ is determined by $f\left(t_{1}\right)$ and $f\left(t_{2}\right), m_{U}$ is determined by $f\left(t_{1}\right)-\varepsilon_{f}$ and $f\left(t_{2}\right)+\varepsilon_{f}$. The value of a task achievement is represented by a triangular fuzzy number $\left(f_{L}, f_{M}, f_{U}\right)$ by using the value of $\left(m_{L}, m_{M}, m_{U}\right)$ as shown in
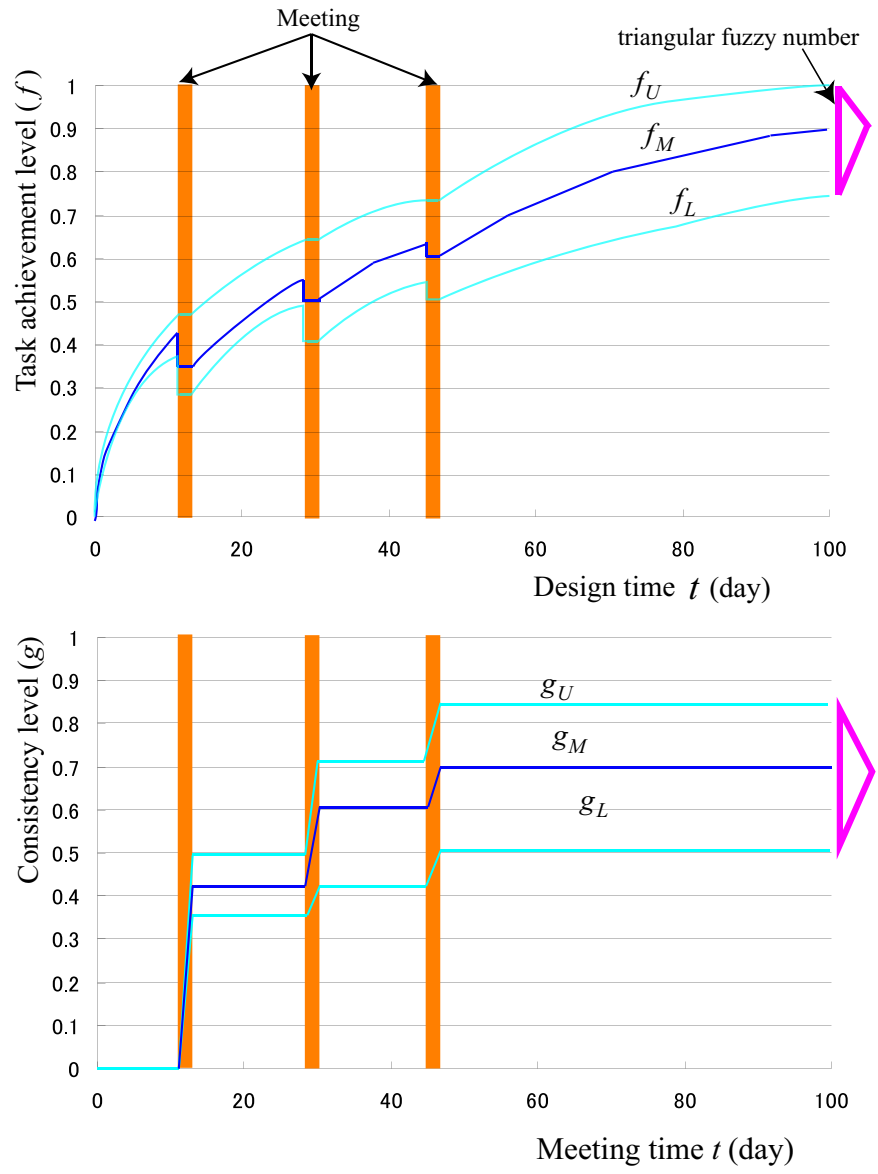

Figure 5. Task consistency and design revision

Figure 4.

In general, a calculation of a triangular fuzzy number can be approximated as a triangular fuzzy number (Kaufmann and Gupta, 1988). When $A=\left(a_{L}, a_{M}, a_{U}\right)$ and $B=\left(b_{L}, b_{M}, b_{U}\right)$ are nonnegative fuzzy numbers, calculations are represented as follows; $A+B=\left(a_{L}+b_{L}, a_{M}+b_{M}, a_{U}+b_{U}\right), A-B=\left(a_{L}-\right.$ $\left.b_{U}, a_{M}-b_{M}, a_{U}-b_{L}\right), A \times B \approx\left(a_{L} b_{L}, a_{M} b_{M}, a_{U} b_{U}\right), A / B \approx$ $\left(\frac{a_{L}}{b_{U}}, \frac{a_{M}}{b_{M}}, \frac{a_{U}}{b_{L}}\right)$.

\subsection{Model of Task Consistency and Design Revision}

A designer acquires a state of knowledge through the design progress from one at a fundamental level to one at an advanced level for a specific task. In collaboration, however, such knowledge acquisition progress is performed under the assumed result of associated tasks or expectation of task consistency. In other words, design achievement may be reduced when the assumption on task consistency is not ensured as a result of design progress. It is empirically rational to assume that the higher the consistency level between tasks, the lesser the 
reduction of design achievement.

Figure 5 shows an outline of an introduced model of a task consistency and design revision. The consistency level increases during each meeting time (lower part of Figure 5). Although the task achievement also increases with a design time, it is reduced during meetings (upper part of Figure 5).

Firstly, Eq. (1) is expanded as the following in order to model the achievement level reduction of task $i$ by a collaborating task $j$ at time $T$;

$$
f_{i}^{\prime}(T)=f_{i}(T)-\Delta Q_{i j}(T)
$$

where, $f_{i}$ and $f_{i}^{\prime}$ are task $i$ 's achievement level before collaboration and after collaboration, respectively. $\Delta Q$ is the reduction of task achievement level.

A consistency level between tasks, $g_{i j}$, is a monotonically increasing function of meeting time. This is modeled as a growth curve by;

$$
g_{i j}(t)= \begin{cases}1-\exp \left(-\frac{\beta}{r_{i j}} \tau_{i j}(t)\right) & :\left(r_{i j} \neq 0\right) \\ 1 & :\left(r_{i j}=0\right)\end{cases}
$$

where, $\tau_{i j}(t)$ is the sum total time of a meeting about task $i$ and task $j$ since the beginning of the design process, and $r_{i j}$ is the degree of dependency between task $i$ and task $j$ that is described in a DSM. In this research, a dependency degree is represented with four numbers, $0,1,3$, or 9 .

The term $\beta$ is a triangular fuzzy number parameter representing meeting efficiency. To define this indistinct parameter, this research empirically assumes that a task consistency increases much more by a meeting within a team than a meeting between different teams. This research determines this parameter based on the case studies as follows; (i) when task $i$ and task $j$ are carried out by the same designer, $\beta=(2.0,2.0,0.0)$. In this case, a meeting means a crosschecking of tasks that is carried out by a designer. (ii) When task $i$ and task $j$ are assigned to different designers, and the number of teams joining the meeting is $n, \beta=(1.0,1.0 / n, 0.0)$.

$\Delta Q$ of Eq. (4) is represented by the following equation;

$$
\Delta Q_{i j}=\alpha r_{i j}\left(1-g_{i j}(t)\right) \cdot \Delta f_{i}(t)
$$

where, $\Delta f_{i}(t)$ is the increment in achievement level of task $i$ from the previous collaboration, $r_{i j}$ is task $i$ 's degree of dependency to task $j$, and $\alpha$ is a tuning parameter. Based on the case analysis of the student design project, this research determines $\alpha=0.05$.

\subsection{Evaluation of Total Acceptability of Design Achievement}

As stated, a design process consists of many tasks each of which corresponds to a part of design achievement. Although some tasks can be done concurrently, the total design process is incomplete without any task. In this sense, a design process

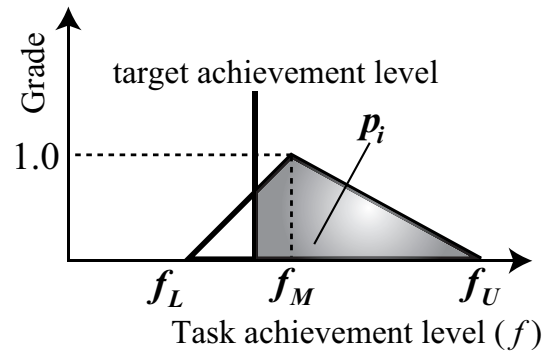

Figure 6. Task achievement probability

can be seen as a serial system of tasks. This research uses a reliability model of a serial system for evaluation of total design achievement. When the probability that task $i$ will achieve an acceptable level is given as $p_{i}$, total acceptability of design achievement $R$ is evaluated by the following equation;

$$
R=100 \prod_{i=1}^{n} p_{i}
$$

where $n$ is the number of tasks.

A task achievement probability $p_{i}$ is given as the proportion of the area of a triangular fuzzy number that exceeds a target achievement level to the area of the entire triangular fuzzy number as shown in Figure 6. When a fuzzy grade at $f_{M}$ is 1.0 , $p_{i}$ is given as;

$$
p_{i}=\frac{2}{f_{U}-f_{L}} A_{i}
$$

where $A_{i}$ is the area of the triangular fuzzy number that exceeds a target achievement level.

\section{FORMULATION OF DESIGN PROCESS PLANNING METHOD}

\subsection{Phases of Design Process Planning}

This research formulates a planning method of a creative and concurrent design process based on a proposed model of design achievement. This method consists of the following four phases; (i) task and iteration identification, (ii) scheduling, (iii) plan evaluation and (iv) plan reconfirmation. Figure 7 shows a flowchart of these phases. This paper mainly focuses on the phases (ii) and (iii).

(i) Task and iteration identification ... This is the first phase of design process planning. Firstly, a design process manager identifies tasks that compose a design process. Possible iterations are identified based on the strength of task dependency. A DSM (Design Structure Matrix) is used to describe a task and a task dependency. Arranging a sequence of tasks and assigning teams by means of a clustering and partitioning algorithm are done to reduce iteration possibility. 


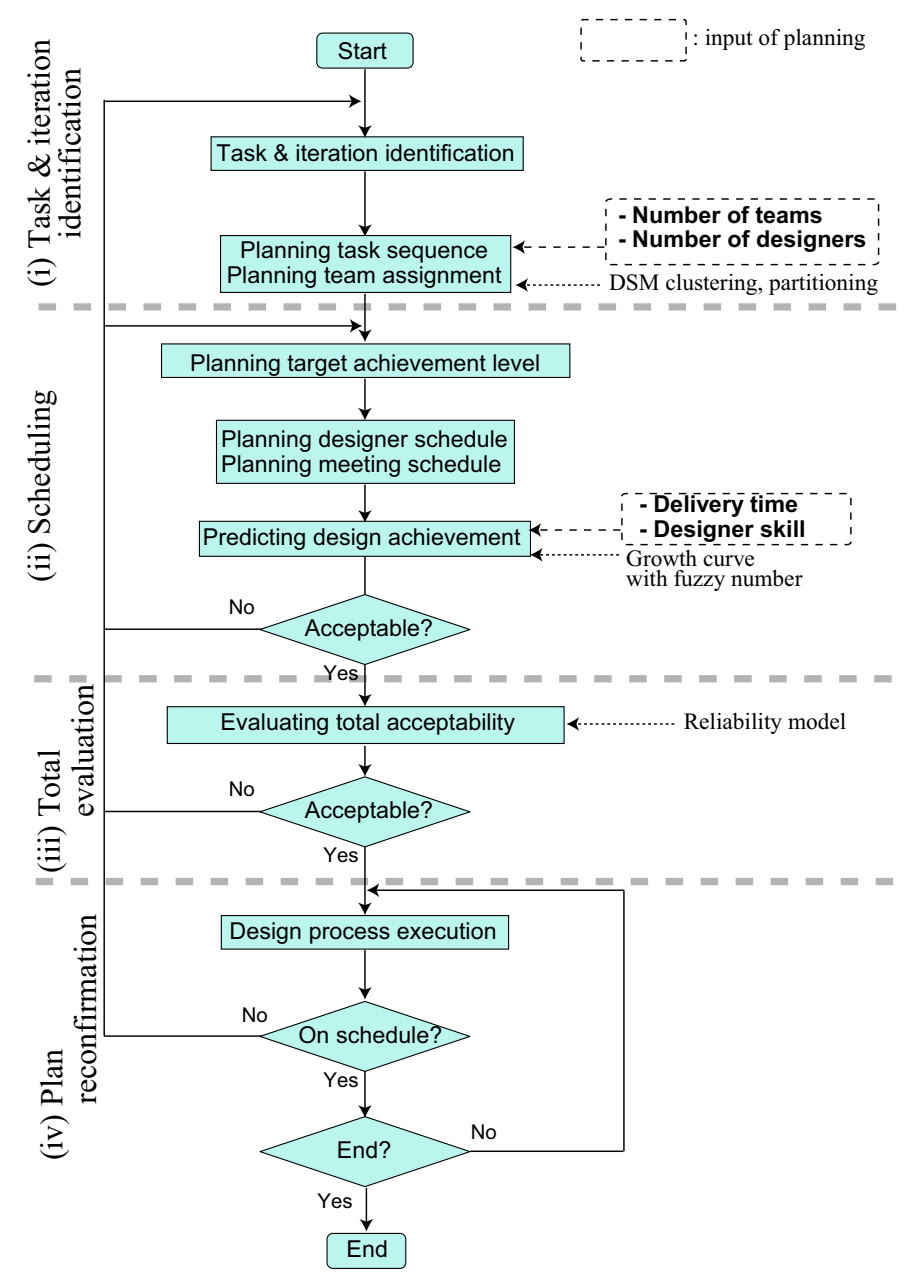

Figure 7. Design process planning

(ii) Task scheduling $\cdots$ A design process manager sets a target achievement level of a task with consideration of the task importance. A manager assigns a skilled designer to an important task, and schedules work days and communication timing so that a task achievement at delivery time satisfies a target achievement level. This scheduling result is usually presented in the form of a Gantt chart. A growth curve model with fuzzy number is used to predict the achievement level of tasks and task consistencies.

(iii) Plan evaluation.. Phase (ii) of the planning process generates some potential alternative plans. In phase (iii), a design process manager should select the most acceptable plan by estimating the total acceptability at delivery time. A reliability model of a serial system is used in this evaluation.

(iv) Plan reconfirmation $\cdots$ In the execution of the design process plan,the actual design achievement often falls short of predictions because of uncertain factors and errors of models. A design process manager should confirm whether or not actual design achievement in on schedule, and modify the planned schedule if necessary.

\subsection{A Prototype System}

In order to verify the capabilities of the proposed method by a case study, its prototype system was implemented in the Java programming language (jdk 1.4.1) running on Windows XP. Figure 8 shows a snapshot of the planning for the formula car design project on the prototype system. Design process planning was carried out as follows. During the first phase, tasks and task dependencies of this project were inputted to the DSM table (Figure 8-(1)). An optimal sequence of tasks and an optimal team assignment were suggested by a partitioning and clustering algorithm. In the case of Figure 8, a clustering algorithm suggested a task assignment plan for four teams. In the second phase, a task schedule and a meeting schedule among the teams were planned by a manager using Gantt charts (Figure 8(2) ). A manager also provided the required delivery time for this project and a target achievement level for each task. Based on these planning data, the system predicted design achievement of each task, and the consistency level between tasks at the delivery time ((Figure 8-(3))). Finally, the total acceptability of a planned design process was calculated ((Figure 8-(4))) so that the manager could evaluate it and explore a better plan.

\subsection{Model Verification}

Firstly, we verify the proposed model of design achievement by comparison between the predicted achievement and the actual achievement result of the OFRAC project. We interviewed OFRAC members in order to collect project data. This project consisted of 27 tasks, which were allocated to four teams. Eleven members were assigned to the design process, which was required to be completed in 84 days. Parameters of a design achievement model were also determined based on the interview. Table 1 shows the initial knowledge level of a designer $f_{0}$, a fuzzy number of task difficulty $m$, and a target achievement level of a task. Uppercase letters denote a designer, with a designer in charge of a task denoted by an asterisk. For example, the difficulty of the "alignment geometry" task is represented by a triangular fuzzy number $m=(0.0175,0.0220,0.0269)$. Designer B takes a charge of the alignment geometry, whose initial level knowledge is 0.1. The collected data are inputted to the planning system. A DSM and a Gantt chart of a task schedule and a meeting schedule are also inputted. Task achievement at the delivery time is predicted based on a growth curve model. The predicted achievement levels are shown in Table 2, where a predicted level is represented by a triangular fuzzy number $\left(f_{L}, f_{M}, f_{U}\right)$.

After the design project was finished, we interviewed the OFRAC team members again in order to determine the 


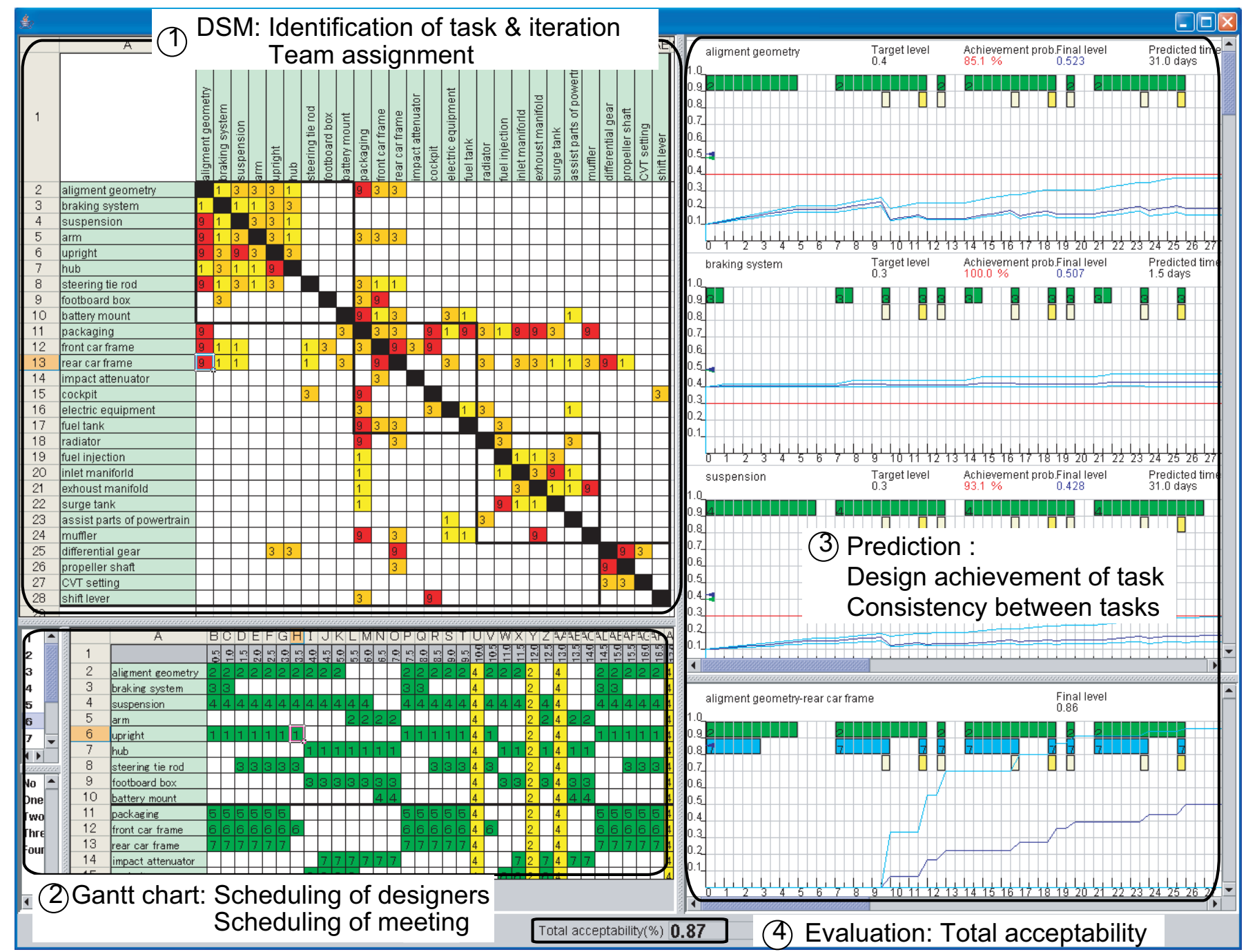

Figure 8. A snapshot of planning a formula car design project

actual achievement level that is shown in the final column of Table 2. Because we asked each OFRAC member to quantify achievement levels on a scale of ten, the value of an actual achievement level is given with a precision of 0.1. All the actual task achievement levels fell within a range of a triangular fuzzy number of a predicted achievement level. The deviation of $f_{M}$ from an actual task achievement was small. Therefore, we conclude that the proposed model of design achievement is valid to predict design achievement.

\subsection{Planning Example}

If the predicted task achievement level is not acceptable, a manager should explore alternative plans. On the planning system, a manager can modify a team assignment on a DSM and modify a schedule on a Gantt chart, and evaluate a new plan by running the prediction again. This subsection shows a simple planning example on the planning system by modifying a task schedule.

Figure 9-(1) shows an actual schedule plan of the OFRAC project (plan 1) during 84 days. A gray rectangle denotes the design time. A yellow rectangle denotes a meeting. The system predicts the task achievement level under the schedule plan. Table 3 gives the prediction of achievement levels as a triangular fuzzy number $\left(f_{L}, f_{M}, f_{U}\right)$, achievement probability $p_{i}$ and a total acceptability of this plan. In plan 1, the achievement levels of some tasks, such as the steering tie rod, packaging and exhaust manifold, are low, while the braking system and the footboard box are overachieved. This prediction result shows that this assignment of design time would be not appropriate. Therefore, a manager changes the task schedule in order to promote better total acceptability. A new schedule plan (plan 2) and variation 
Table 1. Parameters of a design achievement model

\begin{tabular}{|c|c|c|c|c|c|c|c|c|c|c|c|c|c|c|c|c|}
\hline \multirow{2}{*}{ \# } & \multirow{2}{*}{ Task name } & \multicolumn{3}{|c|}{ Task dificulty $(m)$} & \multicolumn{11}{|c|}{ Initial knowledge level of designers $\left(f_{0}\right)$} & \multirow{2}{*}{$\begin{array}{l}\text { Target } \\
\text { level }\end{array}$} \\
\hline & & $m_{L}$ & $m_{M}$ & $m_{U}$ & A & $\mathrm{B}$ & C & $\mathrm{D}$ & $\mathrm{E}$ & $\mathrm{F}$ & $\mathrm{G}$ & $\mathrm{H}$ & $T$ & $\mathrm{~J}$ & $\mathrm{~K}$ & \\
\hline 1 & aligment geometry & 0.0175 & 0.0220 & 0.0269 & 0.0 & $0.1 *$ & 0.1 & 0.0 & 0.0 & 0.0 & 0.0 & 0.0 & 0.3 & 0.0 & 0.0 & 0.4 \\
\hline 2 & braking system & 0.0036 & 0.0182 & 0.0368 & 0.0 & 0.0 & $0.4^{*}$ & 0.0 & 0.0 & 0.0 & 0.0 & 0.0 & 0.2 & 0.0 & 0.0 & 0.4 \\
\hline 3 & suspension & 0.0071 & 0.0104 & 0.0138 & 0.0 & 0.0 & 0.1 & $0.1^{*}$ & 0.0 & 0.0 & 0.0 & 0.0 & 0.2 & 0.0 & 0.0 & 0.3 \\
\hline 4 & arm & 0.0155 & 0.0250 & 0.0347 & 0.0 & $0.1^{*}$ & 0.1 & 0.0 & 0.0 & 0.0 & 0.0 & 0.0 & 0.3 & 0.0 & 0.0 & 0.3 \\
\hline 5 & upright & 0.0128 & 0.0180 & 0.0234 & $0.1^{*}$ & 0.0 & 0.1 & 0.0 & 0.0 & 0.0 & 0.0 & 0.0 & 0.2 & 0.0 & 0.0 & 0.3 \\
\hline 6 & hub & 0.0128 & 0.0193 & 0.0260 & $0.1^{*}$ & 0.0 & 0.1 & 0.0 & 0.0 & 0.0 & 0.0 & 0.0 & 0.2 & 0.0 & 0.0 & 0.3 \\
\hline 7 & steering tie rod & 0.0079 & 0.0140 & 0.0203 & 0.0 & 0.0 & $0.1^{*}$ & 0.0 & 0.0 & 0.0 & 0.0 & 0.0 & 0.2 & 0.0 & 0.0 & 0.3 \\
\hline 8 & footboard box & 0.0111 & 0.0168 & 0.0227 & 0.0 & 0.0 & $0.2 *$ & 0.0 & 0.0 & 0.0 & 0.0 & 0.0 & 0.2 & 0.0 & 0.0 & 0.3 \\
\hline 9 & battery mount & 0.0052 & 0.0260 & 0.0525 & 0.0 & 0.0 & 0.0 & $0.4^{*}$ & 0.0 & 0.0 & 0.0 & 0.0 & 0.0 & 0.0 & 0.0 & 0.4 \\
\hline 10 & packaging & 0.0148 & 0.0224 & 0.0303 & 0.0 & 0.0 & 0.0 & 0.0 & $0.2^{*}$ & 0.1 & 0.1 & 0.0 & 0.0 & 0.0 & 0.0 & 0.3 \\
\hline 11 & front car frame & 0.0104 & 0.0140 & 0.0178 & 0.0 & 0.0 & 0.0 & 0.0 & 0.2 & $0.1^{*}$ & 0.1 & 0.0 & 0.0 & 0.0 & 0.0 & 0.3 \\
\hline 12 & rear car frame & 0.0155 & 0.0210 & 0.0267 & 0.0 & 0.0 & 0.0 & 0.0 & 0.2 & 0.1 & $0.1^{*}$ & 0.0 & 0.0 & 0.0 & 0.0 & 0.3 \\
\hline 13 & impact attenuator & 0.0111 & 0.0168 & 0.0227 & 0.0 & 0.0 & 0.0 & 0.0 & 0.1 & 0.0 & $0.2^{*}$ & 0.0 & 0.0 & 0.0 & 0.0 & 0.4 \\
\hline 14 & cockpit & 0.0215 & 0.0280 & 0.0325 & 0.0 & 0.0 & 0.0 & 0.0 & 0.1 & $0.0^{*}$ & 0.1 & 0.0 & 0.0 & 0.0 & 0.0 & 0.2 \\
\hline 15 & electric equipment & 0.0192 & 0.0280 & 0.0335 & 0.0 & 0.0 & 0.0 & 0.2 & 0.0 & $0.0^{*}$ & 0.0 & 0.0 & 0.0 & 0.0 & 0.0 & 0.2 \\
\hline 16 & fuel tank & 0.0310 & 0.0480 & 0.0584 & 0.0 & 0.0 & 0.0 & 0.0 & 0.0 & 0.0 & $0.0^{*}$ & 0.0 & 0.0 & 0.0 & 0.0 & 0.2 \\
\hline 17 & radiator & 0.0440 & 0.0580 & 0.0732 & 0.0 & 0.0 & 0.0 & 0.1 & 0.0 & 0.0 & 0.0 & $0.2^{*}$ & 0.0 & 0.0 & 0.0 & 0.3 \\
\hline 18 & fuel injection & 0.0083 & 0.0200 & 0.0320 & 0.0 & 0.0 & 0.0 & 0.1 & 0.0 & 0.0 & 0.0 & $0.3^{*}$ & 0.0 & 0.0 & 0.0 & 0.3 \\
\hline 19 & inlet maniforld & 0.0089 & 0.0180 & 0.0271 & 0.0 & 0.0 & 0.0 & 0.1 & 0.0 & 0.0 & 0.0 & 0.2 & $0.1^{*}$ & 0.0 & 0.0 & 0.3 \\
\hline 20 & exhaust manifold & 0.0060 & 0.0120 & 0.0181 & 0.0 & 0.0 & 0.0 & 0.1 & 0.0 & 0.0 & 0.0 & 0.2 & 0.0 & $0.1^{*}$ & 0.0 & 0.3 \\
\hline 21 & surge tank & 0.0060 & 0.0120 & 0.0181 & 0.0 & 0.0 & 0.0 & 0.2 & 0.0 & 0.0 & 0.0 & 0.2 & $0.1^{*}$ & 0.0 & 0.0 & 0.3 \\
\hline 22 & assist parts of pow & 0.0162 & 0.0230 & 0.0301 & 0.0 & 0.0 & 0.0 & 0.0 & 0.0 & 0.0 & 0.0 & $0.2^{*}$ & 0.0 & 0.0 & 0.0 & 0.3 \\
\hline 23 & muffler & 0.0189 & 0.0260 & 0.0334 & 0.0 & 0.0 & 0.0 & 0.1 & 0.0 & 0.0 & 0.0 & 0.1 & 0.0 & $0.1^{*}$ & 0.0 & 0.3 \\
\hline 24 & differenti & 0.0192 & 0.0290 & 0.0390 & 0.0 & 0.0 & 0.0 & 0.0 & 0.0 & 0.0 & 0.0 & 0.0 & 0.0 & 0.0 & $0.1^{*}$ & 0.2 \\
\hline 25 & propeller shaft & 0.0192 & 0.0290 & 0.0390 & 0.0 & 0.0 & 0.0 & 0.0 & 0.0 & 0.0 & 0.0 & 0.0 & 0.0 & 0.0 & $0.1^{*}$ & 0.3 \\
\hline 26 & CVT setting & 0.0043 & 0.0102 & 0.0162 & 0.0 & 0.0 & 0.0 & 0.0 & 0.0 & 0.0 & 0.0 & 0.0 & 0.0 & 0.0 & $0.1^{*}$ & 0.2 \\
\hline 27 & shift lever & 0.0521 & 0.0640 & 0.0733 & 0.0 & 0.0 & 0.0 & 0.0 & 0.0 & 0.0 & 0.0 & 0.0 & 0.0 & 0.0 & $0.0^{*}$ & 0.3 \\
\hline
\end{tabular}

*: a designer in charge of a task

Table 2. Comparison between predicted values and actual results of task achievement level

\begin{tabular}{|c|c|c|c|c|c|}
\hline \multirow{2}{*}{ \# } & \multirow{2}{*}{ Task name } & \multicolumn{4}{|c|}{ Achievement level } \\
\hline & & $f_{L}$ & $f_{M}$ & $f_{U}$ & actual results \\
\hline 1 & aligment geometry & 0.26 & 0.52 & 0.76 & 0.5 \\
\hline 2 & braking system & 0.42 & 0.51 & 0.61 & 0.5 \\
\hline 3 & suspension & 0.23 & 0.43 & 0.60 & 0.4 \\
\hline 4 & arm & 0.16 & 0.42 & 0.55 & 0.4 \\
\hline 5 & upright & 0.18 & 0.40 & 0.62 & 0.4 \\
\hline 6 & hub & 0.24 & 0.50 & 0.62 & 0.5 \\
\hline 7 & steering tie rod & 0.15 & 0.32 & 0.47 & 0.3 \\
\hline 8 & footboard box & 0.31 & 0.49 & 0.63 & 0.5 \\
\hline 9 & battery mount & 0.41 & 0.52 & 0.67 & 0.5 \\
\hline 10 & packaging & 0.20 & 0.28 & 0.69 & 0.3 \\
\hline 11 & front car frame & 0.13 & 0.32 & 0.53 & 0.3 \\
\hline 12 & rear car frame & 0.12 & 0.28 & 0.60 & 0.3 \\
\hline 13 & impact attenuator & 0.39 & 0.50 & 0.58 & 0.5 \\
\hline 14 & cockpit & 0.15 & 0.31 & 0.46 & 0.3 \\
\hline 15 & electric equipment & 0.14 & 0.32 & 0.38 & 0.3 \\
\hline 16 & fuel tank & 0.11 & 0.32 & 0.49 & 0.3 \\
\hline 17 & radiator & 0.33 & 0.42 & 0.64 & 0.4 \\
\hline 18 & fuel injection & 0.38 & 0.50 & 0.62 & 0.5 \\
\hline 19 & inlet maniforld & 0.17 & 0.38 & 0.52 & 0.4 \\
\hline 20 & exhaust manifold & 0.15 & 0.29 & 0.42 & 0.3 \\
\hline 21 & surge tank & 0.22 & 0.43 & 0.62 & 0.4 \\
\hline 22 & assist parts of powertrain & 0.40 & 0.51 & 0.58 & 0.5 \\
\hline 23 & muffler & 0.18 & 0.29 & 0.52 & 0.3 \\
\hline 24 & differential gear & 0.16 & 0.33 & $\overline{0.54}$ & 0.3 \\
\hline 25 & propeller shaft & 0.21 & 0.37 & 0.48 & 0.4 \\
\hline 26 & CVT setting & 0.18 & 0.33 & 0.44 & 0.3 \\
\hline 27 & shift lever & 0.23 & 0.38 & 0.53 & 0.4 \\
\hline
\end{tabular}

of design time of each task are shown in Figure 9-(2). In this plan, designer $\mathrm{C}$ is assigned more design time for the steering tie rod, while his design time for the braking system and footboard box are reduced. The design time of designer $\mathrm{E}$ who is in charge of packaging, and that of designer $\mathbf{J}$ who is in charge of the exhaust manifold, are augmented. After this new schedule plan is inputted, the system predicts that a total acceptability of plan 2 is better than that of plan 1 because the achievement levels of steering tie rod, packaging and exhaust manifold are promoted, while the reduction in the achievement levels of the braking system, footboard box and so on are not considerable (see Table 3). Based on these prediction results, a manager can make the determination that plan 2 is better than plan 1 .

\section{CONCLUSION}

This paper proposes a new method of design process planning that focuses on quantitative prediction of design achievement. In this method, achievement of individual tasks and their consistency is modeled with a growth curve model that uses fuzzy numbers. The degree of achievement and consistency can be predicted based on the model at any stage of the design process. Alternative design process plans can be compared based on the total acceptability calculated with a form of the reliability model of a serial system. The usefulness of focusing on the progressive nature and consistency of knowledge acquisition is revealed by the application of the process planning system, and 
(1) Schedule of plan 1

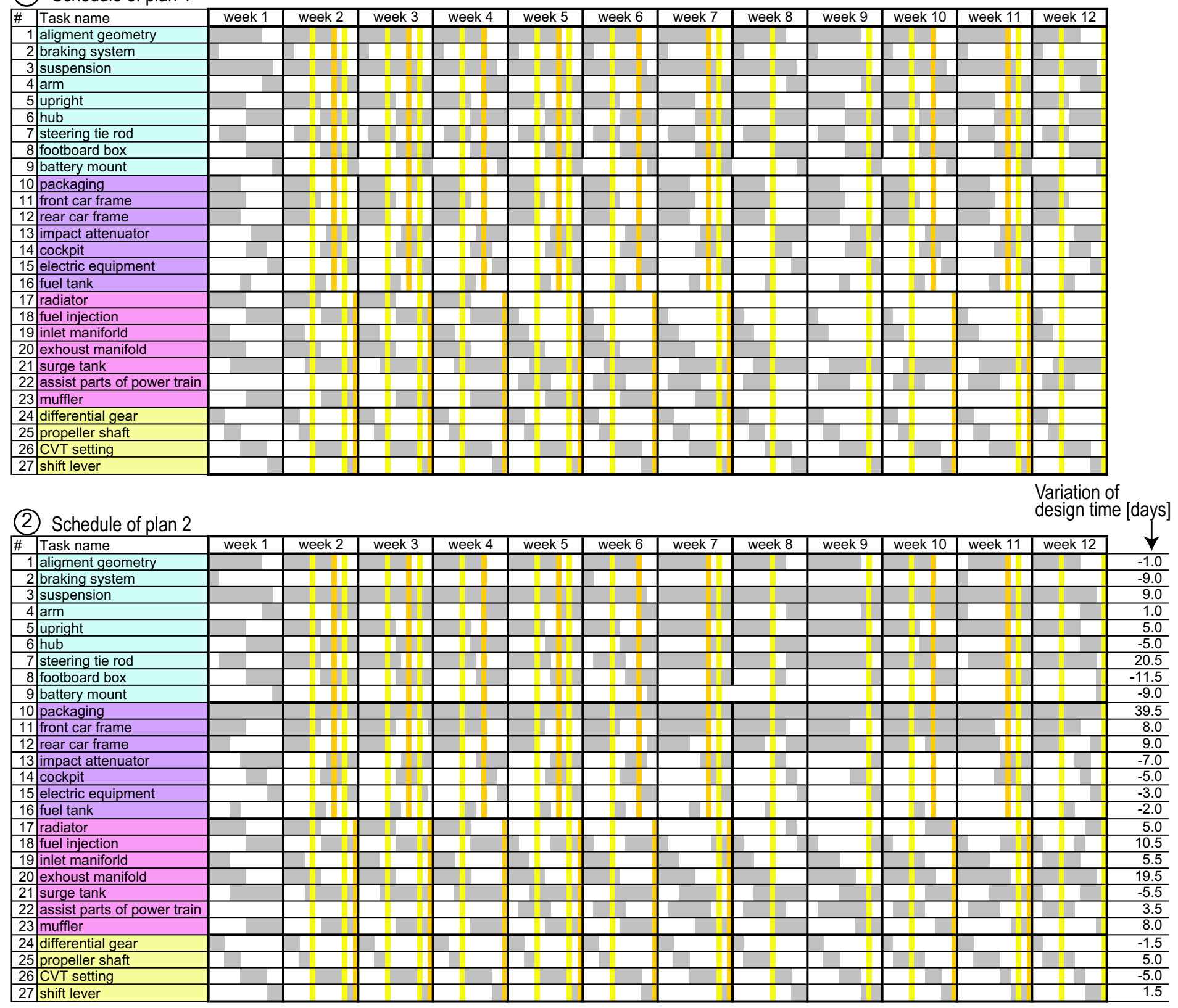

Figure 9. Alternative plans of scheduling

the proposed model is applied to the student design project of a formula racing car. The results of this study indicate the promise of the achievement model and associated planning system.

Our future works include a verification of the generality of a design achievement model, e.g., how sensitive the prediction is to parameter tuning, and what are the limitations of this approach due to factors not considered in the model such as a designer's motivation or limited design resources. However, the verification is not easy because of the inherent indistinctness of the design process. It should be performed through reflective refinement with practical case studies. The meaning of this research is that the proposed method is helpful to explicitly describe the tacit thinking of a design process manager, and to facilitate its verification toward a better planning method. 
Table 3. Comparison between plan 1 and plan 2

\begin{tabular}{|c|c|c|c|c|c|c|c|c|c|c|c|}
\hline \multirow{2}{*}{ \# } & \multirow{2}{*}{ Task name } & \multirow{2}{*}{$\begin{array}{c}\text { Target } \\
\text { level }\end{array}$} & \multicolumn{4}{|c|}{ plan 1} & \multicolumn{4}{|c|}{ plan 2} & \multirow{2}{*}{$\begin{array}{c}\text { increment of } \\
p_{i}\end{array}$} \\
\hline & & & $f_{L}$ & $f_{M}$ & $f_{U}$ & $p_{i}$ & $f_{L}$ & $f_{M}$ & $f_{U}$ & $p_{i}$ & \\
\hline 1 & aligment geometry & 0.4 & 0.26 & 0.52 & 0.76 & 0.85 & 0.26 & 0.52 & 0.75 & 0.84 & -0.01 \\
\hline 2 & braking system & 0.4 & 0.42 & 0.51 & 0.61 & 1.00 & 0.40 & 0.43 & 0.46 & 1.00 & 0.00 \\
\hline 3 & suspension & 0.3 & 0.23 & 0.43 & 0.60 & 0.93 & 0.25 & 0.47 & 0.65 & 0.97 & 0.04 \\
\hline 4 & arm & 0.3 & 0.16 & 0.42 & 0.55 & 0.81 & 0.16 & 0.44 & 0.57 & 0.83 & 0.02 \\
\hline 5 & upright & 0.3 & 0.18 & 0.40 & 0.62 & 0.85 & 0.19 & 0.45 & 0.66 & 0.90 & 0.04 \\
\hline 6 & hub & 0.3 & 0.24 & 0.50 & 0.62 & 0.97 & 0.22 & 0.45 & 0.57 & 0.93 & -0.04 \\
\hline 7 & steering tie rod & 0.3 & 0.15 & 0.32 & 0.47 & 0.59 & 0.19 & 0.47 & 0.65 & 0.90 & 0.31 \\
\hline 8 & footboard box & 0.3 & 0.31 & 0.49 & 0.63 & 1.00 & 0.28 & 0.40 & 0.51 & 0.98 & -0.02 \\
\hline 9 & battery mount & 0.4 & 0.41 & 0.52 & 0.67 & 1.00 & 0.40 & 0.43 & 0.47 & 1.00 & 0.00 \\
\hline 10 & packaging & 0.3 & 0.20 & 0.28 & 0.69 & 0.77 & 0.21 & 0.37 & 0.90 & 0.93 & 0.16 \\
\hline 11 & front car frame & 0.3 & 0.13 & 0.32 & 0.53 & 0.61 & 0.14 & 0.37 & 0.59 & 0.74 & 0.13 \\
\hline 12 & rear car frame & 0.3 & 0.12 & 0.28 & 0.60 & 0.59 & 0.13 & 0.35 & 0.69 & 0.76 & 0.17 \\
\hline 13 & impact attenuator & 0.4 & 0.39 & 0.50 & 0.58 & 0.99 & 0.35 & 0.43 & 0.51 & 0.80 & -0.20 \\
\hline 14 & cockpit & 0.2 & 0.15 & 0.31 & 0.46 & 0.95 & 0.12 & 0.23 & 0.37 & 0.75 & -0.20 \\
\hline 15 & electric equipment & 0.2 & 0.14 & 0.32 & 0.38 & 0.92 & 0.12 & 0.26 & 0.31 & 0.74 & -0.18 \\
\hline 16 & fuel tank & 0.2 & 0.11 & 0.32 & 0.49 & 0.90 & 0.09 & 0.26 & 0.42 & 0.80 & -0.10 \\
\hline 17 & radiator & 0.3 & 0.33 & 0.42 & 0.64 & 1.00 & 0.38 & 0.54 & 0.75 & 1.00 & 0.00 \\
\hline 18 & fuel injection & 0.3 & 0.38 & 0.50 & 0.62 & 1.00 & 0.42 & 0.59 & 0.73 & 1.00 & 0.00 \\
\hline 19 & inlet maniforld & 0.3 & 0.17 & 0.38 & 0.52 & 0.78 & 0.19 & 0.43 & 0.59 & 0.88 & 0.10 \\
\hline 20 & exhaust manifold & 0.3 & 0.15 & 0.29 & 0.42 & 0.41 & 0.19 & 0.43 & 0.60 & 0.88 & 0.47 \\
\hline 21 & surge tank & 0.3 & 0.22 & 0.43 & 0.62 & 0.92 & 0.20 & 0.39 & 0.58 & 0.87 & -0.05 \\
\hline 22 & assist parts of power train & 0.3 & 0.40 & 0.51 & 0.58 & 1.00 & 0.42 & 0.55 & 0.63 & 1.00 & 0.00 \\
\hline 23 & muffler & 0.3 & 0.18 & 0.29 & 0.52 & 0.63 & 0.21 & 0.39 & 0.63 & 0.89 & 0.26 \\
\hline 24 & differential gear & 0.2 & 0.16 & 0.33 & 0.54 & 0.97 & 0.15 & 0.31 & 0.51 & 0.96 & -0.01 \\
\hline 25 & propeller shaft & 0.3 & 0.21 & 0.37 & 0.48 & 0.82 & 0.24 & 0.46 & 0.58 & 0.96 & 0.14 \\
\hline 26 & CVT setting & 0.2 & 0.18 & 0.33 & 0.44 & 0.99 & 0.17 & 0.29 & 0.39 & 0.96 & -0.03 \\
\hline 27 & shift lever & 0.3 & 0.23 & 0.38 & 0.53 & 0.88 & 0.25 & 0.42 & 0.58 & 0.96 & 0.08 \\
\hline \multicolumn{3}{|c|}{ Total acceptability } & & & & 0.87 & & & & 4.64 & \\
\hline
\end{tabular}

\section{ACKNOWLEDGMENTS}

We thankfully acknowledge all OFRAC members for their contribution to the case analysis and study.

\section{REFERENCES}

Chao, L. P. and Ishii, K., 2003, Design Process Error-proofing: Development of Automated Error-proofing Information Systems, Proceedings of ASME 2003 International Design Engineering Technical Conferences \& Computers In Engineering Conference, DETC2003/DAC-48786.

Chao, L. P. and Ishii, K., 2004, Design Process Errorproofing: Project Quality Function deployment, Proceedings of ASME 2004 International Design Engineering Technical Conferences \& Computers In Engineering Conference, DETC2004-57772.

Chao, L. P. and Ishii, K., 2007, Design Process Error-proofing: Failure Modes and Effects Analysis of the Design Process, Journal of Mechanical Design, Transactions of the ASME, Vol. 129, No. 5, pp. 491-501.

Cho, S. H. and Eppinger, S. D., 2005, A Simulation-Based Process Model for Managing Complex Design Projects, IEEE Transactions on Engineering Management, Vol. 52, No. 3, pp. 316-328.

Clarkson, P. J. and Hamilton J. R., 2000, 'Signposting', A Parameter-driven Task-based Model of the Design Process,
Research in Engineering Design, Vol. 12, No. 1, pp. 18-38.

Eppinger, S. D., 1994, A Model-based Method for Organizing Tasks in Product Development, Research in Engineering Design, Vol. 6, No. 1, pp. 1-13.

Student Formula SAE Competition of Japan, http://www.jsae.or.jp/formula/. [Accessed 27th January 2008].

Marca, D. A., 1988, IDEFO/SADT Business Process and Enterprise Modeling, Eclectic Solutions Corp.

Ichikawa, M., 1990, Reliability Engineering, Shokabo Pub. Co. (in Japanese)

Jarrett, J. P., Clarkson, P. J. and Dawes, W. N., 2002, Improving Turbomachinery Design Process management, Proceedings of ASME 2002 International Design Engineering Technical Conferences \& Computers In Engineering Conference, DETC2002/DTM-34015.

Kaufmann, A. and Gupta, M. M., 1988, Fuzzy Mathematical Models in Engineering and Management Science, Elsevier Science Pub. Co.

Melo, A. F. and Clarkson, P. J., 2002, Planning and Scheduling based on an Explicit Representation of the State of the Design, Proceedings of ASME 2002 International Design Engineering Technical Conferences \& Computers In Engineering Conference, DETC2002/DTM-34008.

Nomaguchi, Y., Tsutsumi, D. and Fujita, K., 2007, Design Process Planning from a Viewpoint of Progressive Nature of 
Knowledge Acquisition, Proceedings of 16th International Conference on Engineering Design (ICED 07), Paper No. 490.

Nonaka, I. and Takeuchi, H., 1995, The Knowledge-Creating Company: How Japanese Companies Create the Dynamics of Innovation, Oxford University Press.

O'Donovan, B., Eckert, C. and Clarkson, P. J., 2004, Simulating Design Processes to Assist Design Process Planning, Proceedings of ASME 2004 International Design Engineering Technical Conferences \& Computers In Engineering Conference, DETC2004-57612.

Ostergaard, K. J. and Summers, J. D., 2007, Resistance Based Modeling of Collaborative Design, Concurrent Engineering: Research and Applications, Vol. 15, No. 1, pp. 21-32.

Schön, D. A., 1982, The Reflective Practitioner - How Professionals Think in Action, Basic Books Inc.

Sakamoto, H. and Fujita, K., 2006, An Essay on Availability of Structural Optimization in the Design Process of Electric Appliances, Proceedings of JSME Millennium Vol. 4, pp. 343344. (in Japanese)

Wynn, D., Clarkson, P. J. and Eckert, C. A., 2005, Model-based Approach to Improve Planning Practice in Collaborative Aerospace Design, Proceedings of ASME 2005 International Design Engineering Technical Conferences \& Computers In Engineering Conference, DETC2005-85297.

Yang, T. G., Ishii, K. and Karandikar, H., 2005, Decision Analysis Approach for Improving Product Development Decision Quality: An Interactive Simulation Game Showcase, Proceedings of ASME 2005 International Design Engineering Technical Conferences \& Computers In Engineering Conference, DETC2005-85598.

Yassine, A. A., Whitney, D. E. and Zambito, T., 2001, Assessment of Rework Probabilities for Simulating Product Development Processes using the Design Structure Matrix (DSM), Proceedings of ASME 2001 International Design Engineering Technical Conferences \& Computers In Engineering Conference, DETC2001/DTM-21693. 